\title{
Influencia en la dosis de diálisis de diferentes flujos de líquido dializante en el paciente tratado con hemodiafiltración on-line o hemodiálisis convencional
}

\author{
Ana Vanesa Fernández Martínez - Laura Pérez Valencia - Javier Fernández Caro Sánchez - María Ruiz \\ Serna, Francisco Horrillo Jiménez - Virginia Caparros Ríos - Francisca García Navarro - M ${ }^{a}$ Victoria \\ Vilar Torres
}

Enfermeras

Centro de hemodiálisis nefroclub carthago. Cartagena. Murcia.

\section{Resumen}

La dosis de diálisis influye sobre la supervivencia del paciente en hemodiálisis, además de influir en la corrección de la anemia, en el estado nutricional y en el control de la tensión arterial, entre otros.

Algunos autores han señalado el flujo del líquido dializante como un factor determinante de la dosis de diálisis del paciente en hemodiálisis de alto flujo y hemodiafiltración.

El objetivo del presente estudio es evaluar el impacto en la eficacia de la diálisis de diferentes flujos de líquido dializante en pacientes tratados con hemodiafiltración on-line postdilucional y hemodiálisis convencional, mediante la medición del volumen convectivo final y del aclaramiento de pequeñas moléculas mediante el Kt.

Correspondencia:

Ana Vanesa Fernández

Centro de hemodiálisis Nefroclub Carthago Cartagena

C/ Budapest parc. 136. Polígono Industrial Cabezo Beaza 30325. Cartagena. Murcia

nefroclubcarthago@gmail.com
En todos los pacientes se realizan 9 sesiones de diálisis consecutivas variando el $\mathrm{Qd}: 3$ sesiones con Qd $500 \mathrm{ml} / \mathrm{min}, 3$ sesiones con Qd $800 \mathrm{ml} / \mathrm{min}$ y 3 sesiones con autoflujo .

De los resultados obtenidos, el Kt se muestra significativamente mayor con Qd $800 \mathrm{ml} / \mathrm{min}(59,69 \pm$ 6,07 litros) con respecto a Qd $500 \mathrm{ml} / \mathrm{min}(56,51$ $\pm 6,33$ litros, $p<0,001)$ y autoflujo $(58,02 \pm 4,89$ litros); así como el Kt del autoflujo mayor $(p<0,001)$ que Qd $500 \mathrm{ml} / \mathrm{min}$.

El aumento del flujo de líquido dializante conlleva un incremento en la dosis de diálisis en pacientes tratados con hemodiafiltración on-line. El autoflujo consigue un incremento de dosis apreciable con respecto al $Q d$ de $500 \mathrm{ml} / \mathrm{min}$ con un mínimo coste adicional, por lo que debería de considerarse como una medida en un esquema global de tratamiento, que permita la individualización en cada paciente.

PALABRAS CLAVE:

- DOSIS DE DIÁLISIS

- FLUJO LIQUIDO DIALIZANTE

- HEMODIAFILTRACIÓN EN LINEA

- INDIVIDUALIZACIÓN TERAPÉUTICA 


\section{Influence on the dialysis doses of different flow rates of dialysis fluid in patients treated with online haemodiafiltration or conventional haemodialysis}

\section{Abstract}

The dialysis dose affects survival of patients undergoing dialysis, as well as affecting the correction of anaemia, the nutritional state and the control of blood pressure, among others.

Some authors have indicated the dialysis fluid flow rate as a determining factor in the dialysis dose for patients undergoing high-flow haemodialysis and haemodiafiltration.

The aim of this study is to evaluate the impact on the dialysis efficacy of different flow rates of dialysis fluid in patients treated with on-line post-dilution haemodiafiltration and conventional haemodialysis, by measuring the final convective volume and rinse solution volume of small molecules using $\mathrm{Kt}$.

In all patients, 9 consecutive dialysis sessions were carried out varying the Qd: 3 sessions with Qd 500 $\mathrm{ml} / \mathrm{min}, 3$ sessions with $Q d 800 \mathrm{ml} / \mathrm{min}$ and 2 sessions with autoflow.

From the results obtained, Kt is shown to be significantly higher with Qd $800 \mathrm{ml} / \mathrm{min}(59.69 \pm 6.07$ litres) compared to Qd $500 \mathrm{ml} / \mathrm{min}(56.51 \pm 6.33$ litres, $p<0.001)$ and autoflow (58.02 \pm 4.89 litres); and $\mathrm{Kt}$ in autoflow was found to be higher $(p<0.001)$ than Qd $500 \mathrm{ml} / \mathrm{min}$.

The increase in flow rate of dialysis fluid means an increase in the dialysis dose in patients treated with online haemodiafiltration. The autoflow achieves an appreciable increase in dose compared to Qd of 500 $\mathrm{ml} / \mathrm{min}$ with a minimum additional cost, and should therefore be considered as a measure in an overall treatment framework, permitting individualization of treatment for each patient.

\section{KEY WORDS: \\ - DIALYSIS DOSE \\ - DIALYSIS FLUID FLOW RATE \\ - ONLINE HAEMODIAFILTRATION \\ - INDIVIDUALIZATION OF TREATMENT}

\section{Introducción}

La dosis de diálisis influye sobre la supervivencia del paciente en hemodiálisis ${ }^{1,2}$, habiéndose atribuido la infradiálisis como causa principal de la mayor mortalidad de los pacientes en USA en comparación con Europa o Japón ${ }^{3}$. La dosis de diálisis es un buen marcador de diálisis adecuada ${ }^{4}$, no solo como factor aislado sino que influye en la corrección de la anemia ${ }^{5}$, en el estado nutricional ${ }^{6}$ y en el control de la tensión arterial ${ }^{7}$, entre otros. Las recomendaciones actuales de dosis de diálisis de un estudio multicéntrico americano son de un $\mathrm{Kt} / \mathrm{V}$ igual o superior a 1,3 y un PRU superior al $70 \%{ }^{1}$, cifras que son avaladas por las principales guías internacionales (K-DOQI ${ }^{8}$ y guías europeas ${ }^{9}$ ) y nacionales ${ }^{10}$. Por otro lado, el Kt se ha mostrado como un exigente marcador de dosis de diálisis, capaz de discriminar situaciones de infradiálisis incluso con $\mathrm{Kt} / \mathrm{V}$ normall1,12.

Algunos autores ${ }^{13} 14$ han señalado el flujo del líquido dializante ( $Q d)$ como un factor determinante de la dosis de diálisis del paciente en hemodiálisis de alto flujo y hemodiafiltración, cifrando un incremento del Kt/V entre un 5 y un $15 \%$ (un $8 \%$ en nuestra serie) cuando se comparan flujos de 500 y $800 \mathrm{ml} / \mathrm{min}$.

Recientes avances de la tecnología permiten optimizar el flujo del baño en función de las características del dializador, generando de forma automática un autoflujo de $Q d$ relacionado con el flujo sanguíneo ${ }^{15}$.

El objetivo del presente estudio es evaluar el impacto en la eficacia de la diálisis de diferentes flujos de líquido dializante en pacientes tratados con hemodiafiltración (HDF) on-line postdilucional y hemodiálisis convencional, mediante la medición del volumen convectivo final y del aclaramiento de pequeñas moléculas mediante el Kt. 


\section{Material y métodos}

Se trata de un estudio prospectivo sobre población prevalente en hemodiálisis de nuestra área de salud.

\section{Pacientes:}

\section{Criterios de inclusión:}

- Pacientes mayores de 18 años en tratamiento con hemodiálisis convencional o hemodiafiltración on line posdilucional.

\section{Criterios de exclusión:}

- Menos de un mes en programa de hemodiálisis

- Todos los pacientes firman consentimiento informado.

\section{Métodos}

- En todos los pacientes se realizan 9 sesiones de diálisis consecutivas variando el Qd con el siguiente esquema:

- 3 sesiones con Qd $500 \mathrm{ml} / \mathrm{min}$

- 3 sesiones con Qd $800 \mathrm{ml} / \mathrm{min}$

- 3 sesiones con autoflujo (sistema terapeútico 5008 Fresenius Medical Care)

- En todas las sesiones se utiliza el mismo dializador (helixona 1.3-1.6 $\mathrm{m}^{2}$ ), para el que el autoflujo marca un Qd de 1,5 $\mathrm{ml} / \mathrm{min}$ por $\mathrm{ml}$ de flujo sanguíneo efectivo $(Q b)$.

Las 9 sesiones se realizan con el mismo esquema terapeútico con el sistema terapeútico 5008 Fresenius Medical Care. El volumen de sustitución en los pacientes tratados con hemodiafiltración on-line se realiza de forma automatizada ${ }^{16}$ y el Kt se determina mediante dialisancia iónica (OCM).

\section{Variables en estudio:}

Variable primaria: Kt y litros totales de volumen de sustitución

\section{Variables secundarias:}

- Demográficas: Edad, sexo, tiempo de insuficiencia renal terminal (IRT), etiología de IRT.

- Relacionadas con la diálisis: tiempo efectivo de diálisis y flujo sanguíneo efectivo (Qb).
Todos los parámetros se expresan como valor promedio de las tres sesiones de cada periodo.

\section{Análisis estadístico:}

El análisis estadístico se realiza mediante el programa SPSS 13.0 para Windows.

Las variables cuantitativas se expresan como media, desviación estándar y rango. Las variables cualitativas, como frecuencia y porcentaje. El contraste de hipótesis para variables cuantitativas se realiza mediante la t-student y ANOVA.

\section{Resultados}

128 pacientes se reclutan para el estudio (98 con HDF on-line, 30 en HD convencional). Las características basales de los pacientes en estudio se describen en la tabla 1.

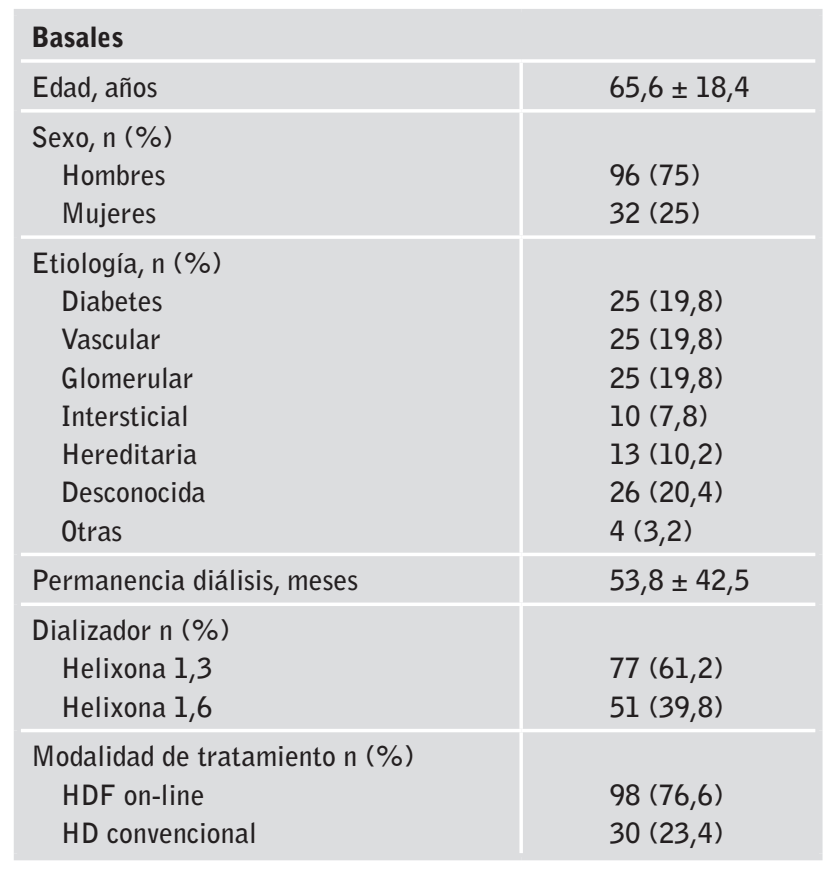

Tabla I. Características basales

Los resultados del Kt y volumen de sustitución en los pacientes en hemodiafiltración on-line aparecen en las figuras 1 y 2 . Se aprecian diferencias significativas en Kt $(p<0,001)$, no así en el el volumen de sustitución.

El Kt se muestra significativamente mayor con Qd 800 $\mathrm{ml} / \mathrm{min}(59,69 \pm 6,07$ litros) con respecto a Qd $500 \mathrm{ml} /$ 
$\min (56,51 \pm 6,33$ litros, $p<0,001)$ y autoflujo $(58,02$ $\pm 4,89$ litros $)$. El Kt del autoflujo se muestra significativamente mayor $(p<0,001)$ que $Q d 500 \mathrm{ml} / \mathrm{min}$.

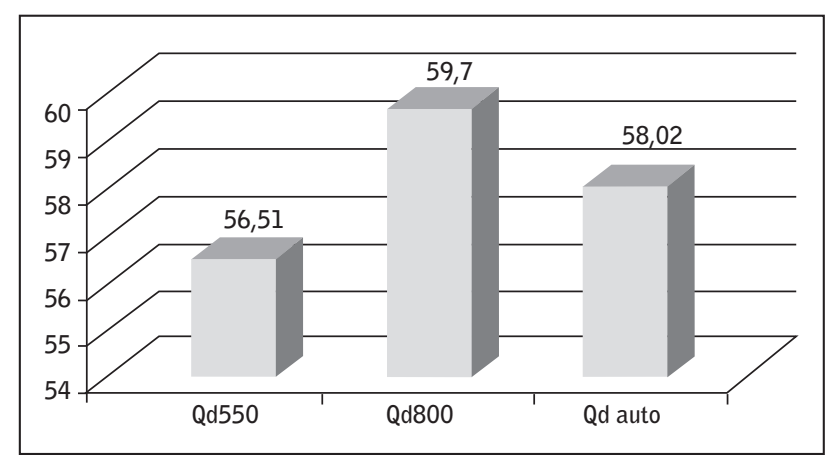

Figura 1: Kt

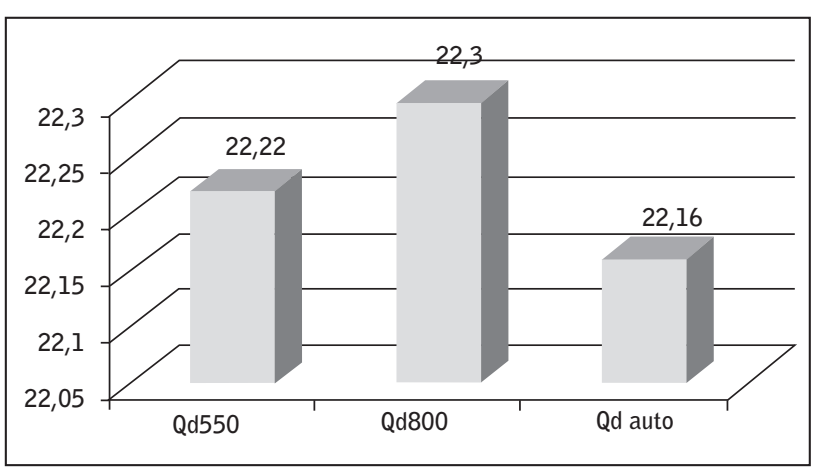

Figura 2. Volumen de sustitución

El Qd auto medio de las tres sesiones fue de 578,54 $\pm 85,53 \mathrm{ml} / \mathrm{min}$.

No se aprecian diferencias significativas en el Kt entre los pacientes en hemodiálisis convencional con Qd 500 $(48,63 \pm 7,01$ litros), Qd auto (48,93 $\pm 6,95$ litros) y Qd 800 (49,47 $\pm 7,46$ litros).

Los datos referentes al Qb y al tiempo efectivo de diálisis se aprecian en la tabla 2. No existen diferencias significativas entre los tres grupos ni en hemodiálisis convencional ni en HDF on-line. Sin embargo, en esta última si se aprecian diferencias en el $\mathrm{Qb}$ en el periodo Qd $500 \mathrm{ml} / \mathrm{min}$, que es mayor $(391,15 \pm 33,95 \mathrm{ml} /$ $\mathrm{min}$ ) cuando se compara con el periodo de $800 \mathrm{ml} /$ $\min (387,12 \pm 27,38 \mathrm{ml} / \mathrm{min}, p=0,038)$ y con el de autoflujo $(390,21 \pm 27,93 \mathrm{ml} / \mathrm{min}, p=0,038)$.

\section{Discusión}

La dosis administrada de diálisis influye en la supervivencia del paciente en hemodiálisis ${ }^{1,2}$. Entre los factores implicados en alcanzar dicha dosis se encuentran el tiempo de la sesión, el flujo sanguíneo efectivo, el aclaramiento de urea del dializador y el flujo del líquido dializante ${ }^{17}$. Como se ha demostrado ${ }^{11,16}$, pequeñas variaciones derivadas de la implicación del personal de enfermería elevan sustancialmente la dosis alcanzada.

En este estudio analizamos el considerado menos importante de los factores mencionados, el flujo del líquido dializante. A la vista de los resultados, en pacientes tratados con hemodiafiltración on-line un $\mathrm{Qd}$ real de $800 \mathrm{ml} / \mathrm{min}$ aporta una mejoría en la dosis de diálisis alcanzada medida por Kt de un 5,6\% con respecto a un flujo de $500 \mathrm{ml} / \mathrm{min}$, resultado que no debe menospreciarse en ningún caso. Por otro lado, el autoflujo propuesto por alguno de los monitores de hemodiálisis del mercado, basado en las características del dializador y en el flujo sanguíneo, si bien se muestra inferior al de 800, aporta un 2,7\% de incremento con respecto al Qd de $500 \mathrm{ml} / \mathrm{min}$, porcentaje que se obtiene con un incremento real de $78 \mathrm{ml} / \mathrm{min}$.

El incremento en la dosis de diálisis en el subgrupo tratado con HDF on-line es menor que el referido por Maduell y cols $(8,6 \%)^{17}$, y otros autores $(8-12 \%)^{18}$, probablemente relacionado con los dializadores utilizados en cada estudio (superficie y KoA), y la diferente

\begin{tabular}{|l|c|c|c|c|c|c|}
\hline & \multicolumn{3}{|c}{ HD CONVENCIONAL } & & \multicolumn{2}{c}{ HDF ON-LINE } \\
\hline & QD 500 & QD AUTO & QD 800 & QD 500 & QD AUT0 \\
\hline Qb, ml/min & $352,09 \pm 51,47$ & $349,52 \pm 49,26$ & $349,69 \pm 47,48$ & $391,15 \pm 33,59$ & $387,12 \pm 27,38$ & $390,21 \pm 33,60$ \\
\hline Tiempo, minutos & $236,53 \pm 13,46$ & $234,58 \pm 14,55$ & $236,91 \pm 11,94$ & $233,13 \pm 6,99$ & $232,21 \pm 6,65$ & $232,70 \pm 6,10$ \\
\hline
\end{tabular}

Tabla 2. Flujo sanguíneo y tiempo efectivo de diálisis 
forma de medición de la dosis de diálisis ( $\mathrm{Kt} / \mathrm{V}$ y Kt). Además, el flujo sanguíneo, factor determinante para el Kt, es significativamente mayor en el grupo de Qd $500 \mathrm{ml} / \mathrm{min}$.

No observamos mejoría significativa en el subgrupo (30 pacientes) de hemodiálisis convencional (incremento de $1,7 \%$ ), donde a los factores anteriormente descritos se podría añadir un flujo sanguíneo más bajo.

Por otro lado, no podemos olvidar la importancia de las medidas que adoptamos en la relación coste-beneficio del proceso de la hemodiálisis. La utilización de un Qd de $800 \mathrm{ml} / \mathrm{min}$ puede incrementar sustancialmente el coste de la sesión con respecto a $500 \mathrm{ml} / \mathrm{min}$ (1 dólar por sesión $\left.{ }^{19}\right)$, cuestión que se minimiza cuando se dispone de autoflujo.

\section{Conclusiones}

El aumento del flujo de líquido dializante conlleva un incremento en la dosis de diálisis, por lo que debe de ser considerado en el caso de pacientes en situación de infra diálisis tratados con hemodiafiltración on-line.

En los casos en los que se disponga de un monitor que permita el autoflujo, este debe ser considerado de elección, ya que consigue un incremento de dosis apreciable con respecto al Qd de $500 \mathrm{ml} / \mathrm{min}$ con un mínimo coste adicional.

En cualquier caso, esta medida debe ser integrada en el marco de un esquema global de tratamiento, que permita la individualización terapeútica en cada paciente.

Recibido: Octubre 2010

Revisado: Noviembre 2010

Modificado: Diciembre 2010

Aceptado: Enero 2011

\section{Bibliografía}

1. Held PJ, Port FK, Wolfe RA, Stannard DC, Carrol CE, Dagirdas JT, Bloembergen WE, Greer JW, Hahim RM: The dose of hemodialysis and patients mortality. Kidney Int 50: 550-556, 1996.
2. Hakim RM, Breyer J, Ismail N, Schulman G: Effects of dose of dialysis on morbidity and mortality. Am J Kidney Dis 23:661-669, 1994.

3. Held PJ, Brunner F, Okada M, García JR, Port FK, Gaylin DS: Five years survival for end stage renal disease patients in the United States, Europe and Japan, 1982-1987. Am J Kidney Dis 15: 451-457, 1990.

4. Maduell F. Dosis de hemodiálisis: condición sine qua non de diálisis adecuada. Nefrología 19 (Sup 4): 51-53, 1999.

5. Ifudu 0, Feldman J, Friedman EA: The intensity of hemodialysis and the response to erithropoietin in patients with end-stage renal disease. N Engl J Med 334: 420-425, 1996.

6. Burrowes DD, Lyons $T A$, Kafman AM, Levin NW: Improvement in serum albumin with adequate hemodialysis. J Renal Nutr 3: 171-176, 1993.

7. Salem MM, Bower J: Hypertension in hemodialysis population: any relation to one-year survival. Am J Kidney Dis 28: 737-740, 1996.

8. NKF-DOQI Hemodialysis Adequacy Work Group Memberchip. Guidelines for hemodialysis adequacy. Am J Kidney Dis 30 (Suppl 2): S22-S63, 1997.

9. European Best Practice Guidelines for Haemodialysis. Nephrol Dial Transplant 17 (Supplement 7): 1721, 2002.

10. Maduell F, García M, Alcazar R: Dosificación y adecuación del tratamiento dialítico. Guías SEN. Guías de centros de hemodiálisis. Nefrología 26 (Suppl. 8): 15-21, 2006.

11. Fernández AV, Soto $S$, Arenas M y cols. Estudio comparativo de la dosis de diálisis medida por dialisancia ionica (Kt) y por Kt/V. 21 Rev Soc Esp Enferm Nefrol 2009;12 (2):97-102.

12. Fernández AV, Pereira MS, Vilar MV y cols. Kt como indicador de dosis de diálisis en una unidad de hemodiálisis. Estudio prospectivo. En: Libro de comunicaciones del XXXIV Congreso de la sociedad Española de Enfermería Nefrologica; Pamplona 2009: 163-167. 
13. Maduell F, García H, Navarro V y Calvo C. Influencia del líquido de diálisis y de la hemodiafiltración sobre la eficacia de la diálisis. Nefrología 16:347352. 1996.

14. Vicente JP, Belchi F, Navarro C y cols. Influencia del aumento del flujo del líquido dializante sobre la eficacia de la diálisis. XXVI Congreso de la SEDEN. 2001.

15. Manual del usuario sistema terapeútico 5008. Fresenius Medical Care.

16. Fernández $A V$, Soto $S$, Arenas $M$ y cols. Comparación de infusión automatica respecto a manual en hemodiafiltración on line posdilucional. Rev Soc Esp Enferm Nefrol 2010;13 (1): 17-22.
17. Maduell F, Navarro V: Medida y control de la eficacia en hemodiálisis. Diálisis adecuada. Monitorización contínua. En Tratado de Hemodiálisis Jofré $\mathrm{R}$, López Gómez JM, Luño J, Pérez García R, Rodríguez Benítez P (eds). Editorial Médica JIMS S.L., Barcelona, pgs 243-270, 2006.

18. Collins A, Liao M, Umen A: High-efficiency treatments using conventional equipment. En Hemodialysis highefficiency treatments. Churchill Livingstone Inc, pp 91-104, 1993.

19. Hootkins R: Dialysate flow rate and dialyzer urea clearence. Semin Dial 8:53; 1995. 